IN brief

\section{Boston quartet revamps biopsies}

Dana-Farber Cancer Institute, Brigham and Women's Hospital, Boston Children's Hospital and the Broad Institute, all in Boston, have launched the Joint Center for Cancer Precision Medicine, headquartered at Dana-Farber. The focus of this clinical cancer genomics center will be on sharing the partners' huge resources in sequencing and new molecular profiling tools to help customize patients' treatment. The initiative will see the creation of a computational biology working group spread across the partner institutions including biologists, bioinformaticians and software designers to develop algorithms aimed at interpreting genome sequencing data. The Joint Center is focused on more than cancer sequencing, says Barrett Rollins, Dana-Farber's CSO. The pathology component is key to the project. Using sequencing, the Center will start with a well-characterized patient population, Rollins says, then follow disease progression and the effects of experimental drugs by watching how cancers respond or become resistant over time. The only way to do that, Rollins says, is to analyze tissue with advanced immunohistochemistry, sequencing, epigenetic analyses or proteomic profiling during treatment. "This is not part of routine clinical care. It's not even part of routine clinical research for the most part," he says. The pathology techniques themselves aren't new, said Rollins, but the operational processes required to support multiple biopsies in a research capacity are. If this approach is successful, the center would "absolutely want to collaborate with as many groups as were interested." Funding for the initiative is from local philanthropy.

\section{IN their words}

\footnotetext{
"Are universities checking to make sure that students have a support network at home to help them cope with bad news? And are the students really prepared to assess a risk that might not become important to them until long after the course is over?" Shawneequa Callier, a bioethics specialist at George Washington University in Washington, DC, questions the practice of offering free genome sequencing as a teaching tool at universities. The Mount Sinai School of Medicine started offering whole genome sequencing to medical and graduate students last fall. (New Scientist, 9 January 2014)

"To those seniors who really do want one [penis pump], just buy it yourself. You don't need to send the bill to your fellow Americans." Ben Domenech of the Heartland Institute, upon hearing that Medicare overpaid by a factor of two for the over 400,000 claims for penis pumps approved from 2006 to 2011. (Huffington Post Business, 13 January 2014)
}

In this protocol, five different agents will be tested against different mutations. The common screening platform means it will be possible to identify and accrue patients with mutations that occur in very small numbers. If a trial arm is testing a compound that could be effective in a small subset of patients, screening may find a mutation that would otherwise not be targeted. Not only those patients who have an appropriate biomarker will be included in one particular arm] Instead, researchers will be aiming for a $60 / 40$ split, with the majority of patients chosen as appropriate and specific for a particular therapy in each of the trial arms, but the rest of them not specifically linked to a particular biomarker.

"This is the age of molecular profiling, and we're moving full bore into that era," says Jeff Abrams of the National Cancer Institute (NCI). "We want these meds to get to patients, and with this trial we can reach out to the community." By community, he is alluding not only to the formal cancer trials network overseen by NCI but also SWOG, one of the largest cancer clinical trials cooperative groups in North America. Patients with advanced-stage squamous-cell lung cancer who seek to enroll will have access to more than 300 clinical sites across North America, all following the same protocol and thus improving patient access to the several experimental therapies being evaluated.

Because the master protocol will be "biomarker driven," says the other protocol co-chair, Vali Papademitrakopoulou of the University of Texas MD Anderson Cancer Center in Houston, "all patients can be eligible" and "no patient should be wasted," she says. For a drug to run in the trial, the one stipulation is that it has its own "suitable and analytically validated biomarker." Each treatment arm is independent of the others within the master protocol, but they each accrue appropriate numbers of patients, all with advanced-stage disease, meaning the experimental treatments are being considered as "second-line therapies."

This first trial for squamous cell lung cancer following the master protocol will aim to enroll between 500 and 1,000 patients a year into four to six arms that will open simultaneously, Papademitrakopoulou says.
As the trial continues, it will become possible to "set up combinations," whereas candidate drugs that are seen as not meeting end points may be dropped. Each of the arms within the master protocol is "totally separate," according to Roy Herbst of Yale Cancer Center in New Haven, who co-chairs the protocol. "So we won't be comparing companies' drugs." Instead, each candidate drug will be compared to the same standard-of-care control arm. Even so, he adds, "We'll have the biggest data set to see which antigens drive the [lung cancer] process."

As the steering committee labored through much of the past year to transform the master protocol concept into reality, its members realized that they "had to choose drugs that were ready" for inclusion in phase 2 clinical trials, Herbst says. Once added to the protocol, each of the treatments will be evaluated on the basis of "progressfree survival" over three months, with each arm powered to detect a twofold increase, says statistician Mary Redman of the Fred Hutchinson Cancer Research Center in Seattle. For phase 3, the primary outcome shifts to overall survival over a median of eight months, and each arm is expected to include about 300 patients, she says.

Other companies were contacted and their candidate treatments considered before the committee whittled its list to five, Herbst says. However, among other promising treatment categories, none of the companies developing monoclonal antibody inhibitors of PD-1 was willing to participate, in part, because several such products are expected to gain FDA approval soon and thus are further along than would be appropriate for evaluation under the new protocol.

That sketch of the treatment-selection process hints at the looming challenges to be faced in implementing this new vision for conducting clinical trials. Not all candidate drugs fit the model, not all companies will want to join in such a complicated, multipartnered enterprise, and patients, too, can be expected to balk over some of its complexities. "This is not easy and could be rough because we've not done it before; it takes pioneers," says FDA's Woodcock. "But there's an ethical imperative that we try this because it offers the best hope for patients."

Jeffrey L Fox Washington, DC 